\title{
Predicting the Curvature of the Cosmos, and Point of Volume Contraction in a Big Bounce Scenario
}

\author{
Christopher Pilot \\ Retired, Maine Maritime Academy, Castine, ME, USA \\ Email: chris.pilot43@gmail.com
}

How to cite this paper: Pilot, C. (2021) Predicting the Curvature of the Cosmos, and Point of Volume Contraction in a Big Bounce Scenario. International Journal of Astronomy and Astrophysics, 11, 265-278. https://doi.org/10.4236/ijaa.2021.112014

Received: April 16, 2021

Accepted: June 18, 2021

Published: June 21, 2021

Copyright $\odot 2021$ by author(s) and Scientific Research Publishing Inc. This work is licensed under the Creative Commons Attribution International License (CC BY 4.0).

http://creativecommons.org/licenses/by/4.0/

\begin{abstract}
Based on the latest Planck surveys, the universe is close to being remarkably flat, and yet, within observational error, there is still room for a slight curvature. If the curvature is positive, then this would lead to a closed universe, as well as allow for a big bounce scenario. Working within these assumptions, and using a simple model, we predict that the cosmos may have a positive curvature in the amount, $\Omega_{0}=1.001802$, a value within current observational bounds. For the scaling laws associated with the density parameters in Friedmann's equations, we will assume a susceptibility model for space, where, $\overline{\chi(a)}$, equals the smeared cosmic susceptibility. If we allow the $\overline{\chi(a)}$ to decrease with increasing cosmic scale parameter, " $a$ ", then we can predict a maximum Hubble volume, with minimum CMB temperature for the voids, before contraction begins, as well as a minimum volume, with maximum $\mathrm{CMB}$ temperature, when expansion starts. A specific heat engine model for the cosmos is also entertained for this model of a closed universe.
\end{abstract}

\section{Keywords}

Cosmic Curvature, Closed Universe, Cyclic Universe, Heat Engine Model for Universe, Big Bounce, Susceptibility Model for Universe, Carnot Cycle Model for Cosmos, Causal Isotropy in CMB Temperature

\section{Introduction}

Models for a cyclic universe and big bounce, versus big bang, scenario, for the cosmos are once again coming into vogue [1] [2] [3] [4] [5]. They bypass some of the long standing problems in cosmology. Among them we include the cosmic volume singularity problem, and horizon problem, i.e., coming up with an explanation for the causal isotropy in $\mathrm{CMB}$ temperature. Big bounce scenarios 
can also explain the smoothness and relative flatness of the universe, allow for a universe without "edges", and avoid the multiverse problem. There are many other reasons. Some good reviews for big bounce, versus big bang models, are given in references [6] [7] [8].

Many such models exist, some of which are very exotic [9] [10] [11] [12] [13]. Some rely on a mechanism whereby the total energy density in Friedmann's equation starts to decrease as the scale parameter, “a”, increases. For a big bounce scenario, we require, namely, that the Hubble parameter, at the point of expansion turning into contraction, equals zero, $H=0$, and furthermore, that, $\dot{H}>0$. A dot over a variable designates a derivative with respect to cosmological time. Unfortunately, this takes us into physics beyond the $\Lambda$ CDM model. In the standard cosmological model, dark energy takes over (dominates) at high " $a$ " values, and stays constant. As such, there is no simple way to scale various density components such that, at some future cosmological epoch, the Hubble parameter, $H$, vanishes.

Some time ago we also proposed, and advocated for, a different type of big bounce model [14]. Our big bounce universe was modeled as a thermodynamic heat engine. Specifically, we argued for a Carnot heat engine cycle for the cosmos, where we have isothermal expansion from points $1 \rightarrow 2$ (see the figure below), followed by adiabatic expansion from points $2 \rightarrow 3$, followed by isothermal contraction from points $3 \rightarrow 4$, followed by adiabatic contraction from points $4 \rightarrow$ 1 , bringing us back to our original starting point. The working substance, which experiences the specific volume expansion and contraction, is the $C M B$ radiation which fills the collective voids in the universe, what is seen in WMAP and Planck data. This part which expands, and will later contract, is called the "system". The "surroundings" are collectively the cooler regions (pockets) in the universe which have given up heat initially, and which will later fill with ordinary matter. This part will hardly expand (it may even contract) due to the action of losing heat energy, and eventually gravity will prevent further expansion. Diagrammatically, the four-step process is represented by Figure 1, which is not drawn to scale. It is for representative purposes only. The universe, currently, finds itself somewhere between points, 2, and 3, towards the bottom end near point 3.

In Figure 1, the thermodynamic point 1 represents the minimum volume, the maximum temperature, the maximum internal energy density, the maximum pressure, and the maximum entropy density, for the $\mathrm{CMB}$ radiation, which we currently see in WMAP/Planck temperature maps. Point 3, on the other hand, indicates the maximum volume, the minimum temperature, the minimum internal energy density, and the minimum entropy density, for same. From point 1 to point 2, an amount of heat, $\left|Q_{H}\right|$, is given up by the surroundings to the system, and from point 3 to 4 , heat energy is supplied by the system, $\left|Q_{C}\right|$, and given back to the surroundings.

The advantages of this proposed heat engine model are many. Among the most important [14], we would list, 


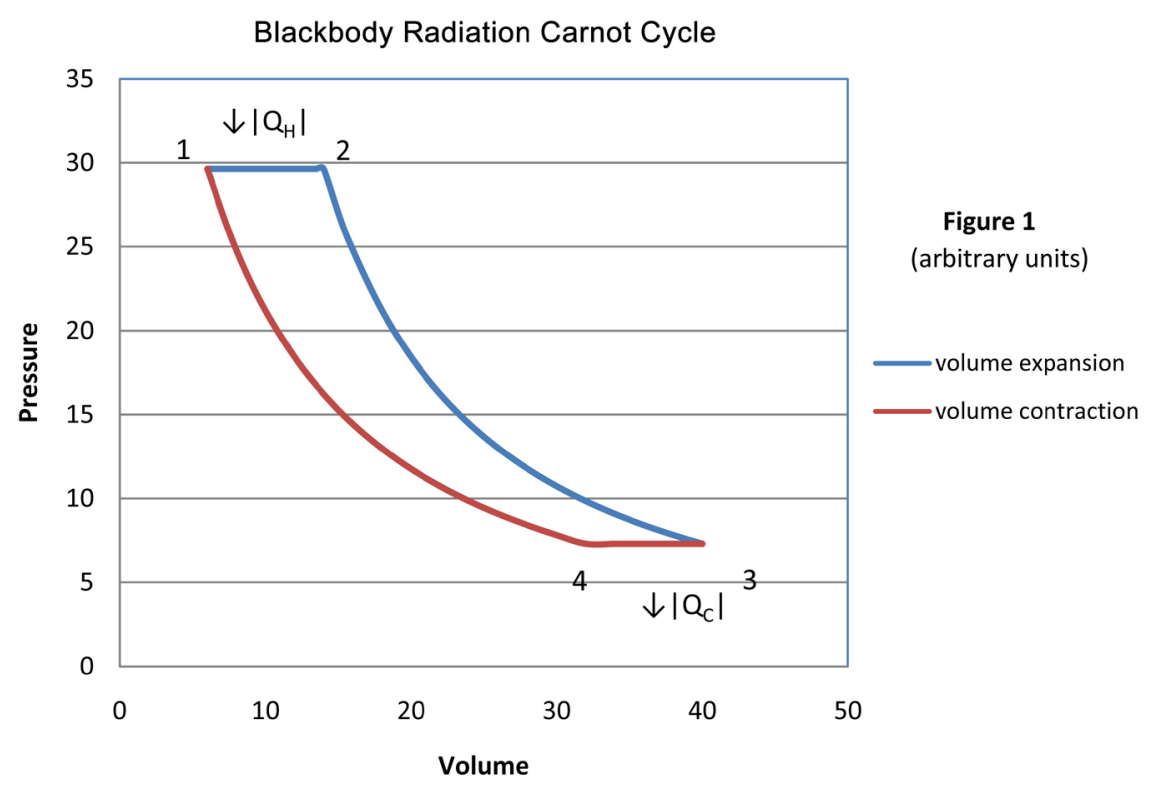

Figure 1. The lines from points 1 to 2 , and from points 3 to 4 , are drawn greatly exaggerated lengthwise in this diagram. They should be drawn almost infinitely close to one other if this figure were to scale. Figure 1 is definitely not to scale in either the $\mathrm{x}$ or $\mathrm{y}$ sense and it given for qualitative, illustrative purposes only.

1) No inflaton field is needed. The isothermal expansion phase from point 1 to point 2 in the diagram above is identified with cosmic inflation, lasting about, $10^{-35}$ seconds.

2) We can explain $\Delta T / T \cong 5 \times 10^{-5}$ at thermodynamic point 2 . The $\mathrm{CMB}$ temperature at this point is estimated to equal, $T_{2}=T_{1}=3.01 \times 10^{27}$ Kelvin .

3) No CMB volume singularity exists at point 1 , as this point has a finite volume, a finite energy density, a finite pressure, and a fixed temperature.

4) We have an actual physical mechanism for evaluating the work done by the universe in expanding, and then contracting. If we were to consider only the process whereby we proceed from point 2 to point 3 and then directly back again to point 2 , then there would be no area enclosed under the pressure versus volume diagram above. In other words no work would be done by the CMB radiation in expansion and contraction. Isothermal expansion, and isothermal contraction, is a necessary input, we believe, to define a certain amount of work done.

5) The universe is cyclic having no beginning nor end in time. It does however have an upper limit in volume, which we call, $V_{3}$, and lower bound in volume, which we designate by, $V_{1}$, to conform to the thermodynamic points illustrated in the diagram, Figure 1 . The pressure and volume refer only to the CMB radiation, which we see today in WMAP and Planck satellite data. It amounts to, $9.153 \times 10^{-5} \rho_{0}$, where $\rho_{0}$ is the current mass density of the universe, given our choice for Hubble constant.

6) Being a closed universe, the cosmos has no "edges", a problem which was already appreciated by Einstein, in 1917. He advocated for a universe without 
boundaries [15] [16], as did Willem de Sitter, Carl Friedrich von Weizsäcker, and George Gamow.

There are other reasons, which were discussed in reference [14], and which will not be repeated here.

As mentioned, it was estimated that, $T_{2}=T_{1}=3.01 \times 10^{27}$ Kelvin, in the isothermal process lasting approximately $10^{-35}$ seconds in going from point 1 to point 2. Moreover, the volume increase in $\mathrm{CMB}$ radiation in this process was estimated to equal a mere, $V_{2} / V_{1} \cong 5.65$, a very modest increase [14] in comparison to the standard inflation scenario. What drives volume expansion in this isothermal process is entropy increase to the voids, a one way street from surroundings to system, making the surroundings cooler and the system hotter, as a consequence. Once the system collective volume increases, there is no heat energy left over for the system to give back to the surroundings. It is in this isothermal phase, specifically, that we have entropy increase to the system, an amount calculated to equal, $S_{2} / S_{1} \cong 5.65$. The entropy density is immense and can be calculated, as well as internal energy density, and pressure, given the estimated temperature. These quantities are all functions of temperature, and temperature only, for blackbody radiation [14]. The surrounding is treated as a reservoir from which heat can be drawn, and if large enough, may not necessarily contract as a consequence.

A natural question arises. Can the CMB temperature at thermodynamic point 3 in the diagram above, $T_{3}$, be estimated? This would indicate the point of contraction for the cosmos as a whole. Knowing the present Hubble radius, we would be able to determine the future Hubble radius, $R_{3}$, where the Hubble constant momentarily vanishes, $H_{3}=0$, and where we have maximum volume, $V_{3}$. We believe the answer is yes, and this is the main thrust of this paper. For that we need to postulate a closed universe, and then predict the curvature in the present cosmos. This is done in Section 2. We will also need a specific mechanism for decreasing the total density parameter in Friedmann's equation. This is done in Section 3, where we introduce a decreasing smeared cosmic susceptibility, $\overline{\chi(a)}$, with increasing scale parameter, which is based on previous published work [17] [18]. We will show explicitly how this feature can cause the universe to eventually contract. Furthermore, we will be in a position to calculate how much longer it will expand given a very crude, but specific one-parameter model. Finally in Section 4, our summary and conclusions are presented.

\section{Friedmann's Equation with Curvature, and Estimating $\Omega_{0}$}

We start with Friedmann's equation with curvature built in. As is well-known, it can be written in the form,

$$
\dot{R}^{2}-(8 \pi G / 3) \rho R^{2}=-k c^{2}
$$

The variable, is the Hubble radius, $G$ equals Newton's constant, and a dot over a quantity designates a derivative with respect to cosmological time. The Friedmann equation shows that a universe that is spatially closed (with $k=+1$ ) has 
negative total "energy": the expansion will eventually be halted by gravity, and the universe will recollapse. Conversely, an unbound model is spatially open $(k=-1)$ and will expand forever. For a flat universe, $k=0$. Equation (2-1) can be rewritten in terms of the Hubble constant, $H \equiv \dot{R} / R$, as,

$$
H^{2}-(8 \pi G / 3) \rho=-k c^{2} / R^{2}
$$

We also know that for a given rate of expansion there is a critical density that will bring the expansion asymptotically to a halt:

$$
\rho_{\text {CRIT }}=3 H^{2} /(8 \pi G)
$$

Therefore, upon rearranging terms, Equation (2-2), can be re-expressed as,

$$
\rho=\rho_{\text {CRIT }}+\left(k c^{2} / R^{2}\right)(3 /(8 \pi G))
$$

We can define a density parameter as the ratio of actual density to critical density. Using Equation (2-4), we find that,

$$
\Omega \equiv \rho / \rho_{\text {CRIT }}=8 \pi G \rho /\left(3 H^{2}\right)
$$

If, $k=0$, then we have a flat universe, and $\Omega=1$. If we have positive curvature, $k=+1$, then the universe is closed, and in this situation, by Equation (2-5), $\Omega>1$. For an open universe, $k=-1$, and we find that $\Omega<1$. From here on in, we will assume a closed universe where, $k=+1$.

With conventional scaling ( $\Lambda \mathrm{CDM}$ model), we have

$$
H^{2}=8 \pi G \rho / 3=H_{0}^{2}\left[\Omega_{R, 0} a^{-4}+\Omega_{\text {MATTER }, 0} a^{-3}+\Omega_{D E, 0}-\left(\Omega_{0}-1\right) a^{-2}\right]
$$

In this equation, " $a$ " is the cosmic scale parameter; we are using the convention where, $a_{0}=1$. The scale parameter is related to the redshift, $Z$, by the equation, $a=T_{0} / T=R / R_{0}=(1+Z)^{-1}$. The temperature, is the CMB temperature, and $R$ is the observable Hubble radius. Hubble's constant can be rewritten as, $=\dot{R} / R=\dot{a} / a$. All subscripts, "0", refer to the present epoch and variables without a subscript refer to a different cosmological epoch. The density parameters, $\Omega_{R, 0}, \Omega_{\text {MATTER, } 0}, \Omega_{D E, 0}$, refer to the current epoch values for radiation, matter (ordinary and dark matter), and dark energy components, respectively.

If we specialize Equation (2-6), to the present epoch, we see that,

$$
\Omega_{R, 0}+\Omega_{\text {MATTER }, 0}+\Omega_{D E, 0}-\left(\Omega_{0}-1\right)=1
$$

We also note that, using Equations (2-5), (2-4) and (2-3), we have

$$
(\Omega-1)=k c^{2} /\left(H^{2} R^{2}\right)
$$

Another way to write Equation (2-8) is to make use of Equation (2-6). We can prove that,

$$
\begin{aligned}
& (\Omega-1)=k c^{2} /\left(H^{2} R^{2}\right) \\
& =\left(k c^{2} /\left(R_{0}^{2} a^{2}\right)\right) H_{0}^{-2}\left(\Omega_{R, 0} a^{-4}+\Omega_{\text {MATTER }, 0} a^{-3}+\Omega_{D E, 0}-\left(\Omega_{0}-1\right) a^{-2}\right)^{-1} \\
& =\left(\Omega_{0}-1\right) /\left(\Omega_{R, 0} a^{-2}+\Omega_{\text {MATTER }, 0} a^{-1}+\Omega_{D E, 0} a^{2}-\left(\Omega_{0}-1\right)\right)
\end{aligned}
$$

This beautiful result will allow us to find the amount of curvature in any 
epoch, given the current value.

In the current epoch, the radiation component equals, $\Omega_{R, 0}=9.153 \mathrm{E}-5$. We assume that, at present, $T_{0}=2.725 \mathrm{Kelvin}$, and we have three species of neutrinos. Our value for $H_{0}$ equals, $67.74 \mathrm{~km} /(\mathrm{s} \cdot \mathrm{Mpc})=6.925 \times 10^{-11} \mathrm{yr}^{-1}$, as estimated by the latest Planck surveys [19] [20] [21]. We know that, $\Omega_{R, 0} h^{2}=4.2 \mathrm{E}-5$, where $h \equiv H_{0} /(100 \mathrm{~km} /(\mathrm{s} \cdot \mathrm{Mpc}))$. Using the Hubble value measured above and solving for $\Omega_{R, 0}$ gives us the value indicated. The radiative contribution is so small that it is typically ignored when discussing the future fate of the universe. However, we will include it in our discussion as it precisely defines our "system" in the thermodynamic heat engine.

For dark energy, the latest estimate is, $\Omega_{D E, 0}=0.6911$, as indicated by the Planck Collaborations [19] [20] [21]. According to the $\Lambda$ CDM model, this does not scale as the universe expands. In the quintessence models, the dark energy component is assumed to barely scale.

We next consider the observed value for, $\Omega_{0}$. This is not exactly equal to unity, but has the value [19] [20] [21],

$$
\Omega_{0}=1.00231_{-0.0054}^{+0.0056}
$$

Within observational error, this value is so close to one, that it is often assumed that it equals precisely unity, as in the $\Lambda \mathrm{CDM}$ model. We will however relax this assumption. We will instead take this value as precise, and claim for the time being, that, $\Omega_{0}=1.00231_{-0.0}^{+0.0}$. In other words, we will not assume flatness, where, $\Omega_{0}=1$. Using our precise value for, $\Omega_{0}$, and Equation (2-8), we find that the Hubble radius equals,

$$
R_{0}=\left(c / H_{0}\right)(0.00231)^{-1 / 2}=2.841 \mathrm{E} 27 \text { meters }
$$

This calculated value is very, very close to another value obtained by entirely different means. That value was, $R_{0}=3.217 \mathrm{E} 27$ meters . It was obtained by relating dark matter to dark energy through a polarization model for space [17] [18]. It is a very precise value, good to three significant figures, because it is based entirely on the present estimates for the density parameters in Friedmann's equation. We will henceforth use this estimate and work backwards to find our predicted value for density curvature. Using Equation (2-8), we find that

$$
\left(\Omega_{0}-1\right)=c^{2} /\left(H_{0}^{2} R_{0}^{2}\right)=1.802 \mathrm{E}-3 \quad \text { (predicted curvature) }
$$

This small value is well within observational bounds as indicated by Equation (2-10).

We next calculate $\Omega_{\text {MATTER }, 0}$ using Equations (2-12), and (2-7). We believe the estimates for, $\Omega_{R, 0}$, and, $\Omega_{D E, 0}$, given above to be quite accurate. The estimate for, $\Omega_{\text {MATTER, } 0}$, is probably not as accurate, as it factors in ordinary matter and dark matter. Using Equation (2-12), we calculate that,

$\Omega_{\text {MATTER }, 0}=1.001802-0.6911-9.153 \mathrm{E}-5=0.3106$. We are now in a position to ask at what point the universe will start to contract. For the universe to start 
contracting, we demand that, $H=0$. Thus, we will attempt to solve Equation (2-6), under the condition that $H=0$ for a particular scale factor, $a_{3}$, corresponding to point 3 in the diagram above, Figure 1. From this equation, for a big bounce from expansion to contraction, the following condition must be satisfied.

$$
\Omega_{R, 0} a_{3}^{-2}+\Omega_{\text {MATTER }, 0} a_{3}^{-1}+\Omega_{D E, 0} a_{3}^{+2}=\left(\Omega_{0}-1\right)
$$

This equation, however, can never be satisfied for, $a_{3}>1$, given the density parameters listed above. This leads us to conclude that a big bounce scenario is impossible with conventional, $\Lambda \mathrm{CDM}$ model, scaling. The third term on the left hand side, the dark energy contribution, makes it impossible. If we believe in a big bounce contraction, and a closed universe, some other mechanism for scaling is required.

\section{Heat Engine Model and Subsequent Contraction}

As mentioned in the introduction, our thermodynamic heat engine model for the cosmos consists of four separate processes, as outlined in our diagram, Figure 1. We have isothermal expansion from point 1 to point 2 , our cosmic inflation phase. This is followed by adiabatic expansion, from point 2 to point 3 . Isothermal contraction from point 3 to point 4 follows, which is our cosmic deflation phase. And finally, to bring us back to our initial starting point, from point 4 to point 1, we have adiabatic contraction. From the voids perspective, heat energy from surroundings to system (the voids) drives an increase in entropy in going from, $1 \rightarrow 2$. This increases the volume of the voids. From, $2 \rightarrow 3$, we have adiabatic expansion driven by a decrease in internal energy density. The entropy stays constant. In going from, $3 \rightarrow 4$, a loss of heat drives volume contraction, with an attendant loss of entropy (given up to the surroundings). And finally from, $4 \rightarrow 1$, volume contraction is caused by an increase in internal energy density. All these variables refer to the CMB radiation seen in the WMAP/ Planck data.

The work done, per cycle, is the enclosed area under the pressure versus volume loop in Figure 1. We calculated this to equal [14],

$$
\begin{aligned}
W_{\text {TOTAL }} & =\left|Q_{H}\right|-\left|Q_{C}\right| \\
& =4 p_{H}\left(V_{2}-V_{1}\right)-4 p_{C}\left(V_{3}-V_{4}\right) \\
& =4 p_{H}\left(V_{2}-V_{1}\right)-4 p_{C}\left(T_{H} / T_{C}\right)^{3}\left(V_{2}-V_{1}\right) \\
& =4 p_{H}\left(V_{2}-V_{1}\right)\left[1-\left(p_{C} / p_{H}\right)\left(T_{H} / T_{C}\right)^{3}\right] \\
& =4 p_{H}\left(V_{2}-V_{1}\right)\left(e_{\text {CARNOT }}\right)
\end{aligned}
$$

In these equations, $\left|Q_{H}\right|$, is the heat energy absorbed by the voids in process 1 $\rightarrow 2$, at CMB temperature, $T_{1}=T_{2}=T_{H}$. The, $\left|Q_{C}\right|$, is the heat lost by the voids to the surroundings in process, $3 \rightarrow 4$, at temperature, $T_{3}=T_{4}=T_{C}$. The $p_{H}$ is the CMB radiative pressure at, $T_{1}=T_{2}=T_{H}$, whereas, $p_{C}$ is the CMB radiative pressure at, $T_{3}=T_{4}=T_{C}$. The $V_{i}$ stands for the radiative volume at thermodynamic point, $i$. And, $e_{\text {CARNOT }}$, stands for the efficiency of this Carnot cycle. It turns out 
[14] that

$$
e_{\text {CARNOT }}=\left(1-T_{C} / T_{H}\right)
$$

We notice that the efficiency is defined just like that for an ideal gas. We keep in mind, however, that we are dealing with CMB blackbody radiation, and only that portion which is visible today in WMAP/ PLANCK satellite data. Because, $T_{C} \ll T_{H}$, this cycle is very close to $100 \%$ efficient. A knowledge of $T_{C}$ is needed to calculate the exact efficiency, as well as the exact amount of work done by this radiation. We estimated $T_{H}$ to equal 3.01E27 Kelvin [14]. The heat transfer from point 1 to point 2 caused a temperature fluctuation decrease from, $\Delta T / T \sim 1$, to, $\Delta T / T \approx 5 \mathrm{E}-5$, which is what we observe today in satellite data.

As seen in Section II, conventional scaling behavior is problematic for proving a big bounce contraction. Modifications to the standard model have to be made. While many big bounce models are interesting, we believe that our heat engine model for the cosmos is particularly straight forward and intuitive. However, what kind of scaling behavior can we assume for the various components making up the total energy density? We are specifically thinking of dark matter and dark energy scaling. We do not believe that dark matter scales like ordinary matter, nor do we accept that dark energy barely scales. Our reasoning is given in references [17] [18]. In those publications, we believe that dark matter and dark energy are actually related, and have an intrinsic origin, the polarization and susceptibility of space. This will contribute totally to dark matter, and partially to dark energy. The details can be found in those works.

What is needed for our purposes are the scaling laws for dark matter, and dark energy. These were found in reference [18] as,

$$
\rho_{D M} / \rho_{D M, 0}=\left(\chi / \chi_{0}\right)\left(K_{0} / K\right) a^{-3}, \rho_{D E} / \rho_{D E, 0}=\left(K_{0} / K\right) a^{-3} \quad(3-3 \mathrm{a}, \mathrm{b})
$$

In these equations, $\chi=\chi(a)$, is the cosmic susceptibility due to macroscopic, gravitational Planck particle dipole formation, and alignment. The relative gravitational permittivity, $K(a)$, is related to $\chi(a)$ through the equation, $K(a)=1-\chi(a)$. We are adopting a Winterberg model for space, where we have a vast assembly (sea) of positive and negative mass Planck particles, which together form an electrically neutral, and massively neutral medium, the vacuum, in the unperturbed state.

In reference [18], we assumed that $\chi(a)$ increases with an increase in cosmological time. This assumption will have to be relaxed in this paper. After a certain point in time, the $\chi(a)$ may actually decrease with increasing cosmological time, i.e., with increasing scale parameter, a. This goes counter to previous thinking. The reason we assumed that $\chi(a)$ must increase with increasing " $a$ " is because ordinary matter had to form before the space surrounding it could be polarized. It is well known that ordinary matter is made up of quarks and leptons, particles which only started to freeze out below, E16 Kelvin (1 Tev) [22] [23] [24] [25]. Before that point, time wise, we presumably only had radiation, and now possibly, Planck particles, which are called planckions by Winter- 
berg. We also believe that atomic matter clumping into ordinary matter (solids, liquids, gases, and plasmas) was necessary before the surrounding space could be polarized. Thus the inception temperature for significant macroscopic susceptibility was probably after recombination, i.e., after, $a \cong 1 / 1100$. Only from that point onwards could solids, liquids, and non-ionized gases form. We assumed specific functions whereby $\chi(a)$ increased with increasing cosmic scale parameter in reference, [18]. However, this will never lead to eventual big bounce contraction. At some point in cosmological time, the $\chi(a)$ must flip, and start to decrease with increasing scale parameter, " $a$ ". We can call the cosmological flip point, $a^{*}$. We are imagining a charging up, and then, discharging process for, $\chi(a)$, much like in a capacitor.

Formalizing this scenario further, let us assume that,

$$
\begin{gathered}
\chi(a)=\left[1-\mathrm{e}^{-a \chi_{0}}\right] a<a^{*} \quad \text { (charging up process) } \\
\chi(a)=\mathrm{e}^{-\lambda a} a \geq a^{*} \quad \text { (discharging process) }
\end{gathered}
$$

The critical epoch of flip is characterized by scale parameter, $a^{*}$. One will note that, $0 \leq \chi(a) \leq 1$, in both instances. This is necessary so that, at every point in cosmological time, $\chi(a)+K(a)=1$, where, $K(a)$, is the relative gravitational permittivity. Equation (3-4a), was an equation that we worked with in reference [18], and assumed an increasing $\chi(a)$ with increasing cosmic scale parameter, " $a$ ". The, $\lambda$, in Equation (3-4b), is new, and is a parameter which needs to be determined, if we accept a decreasing $\chi(a)$ with increasing scale parameter, " $a$ ".

We will assume that the universe is currently in the decay mode with respect to cosmic susceptibility. We found in reference [17], that, $\chi_{0}=\chi\left(a_{0}=1\right)=0.842$. This left us with, $K_{0}=K\left(a_{0}=1\right)=0.158$, such that, $\chi+K=1$, is satisfied. We therefore specialize Equation (3-4b), to the present epoch and demand that,

$$
0.842=\mathrm{e}^{-\lambda 1}
$$

The solution is, $\lambda=0.1720$. Using this value in Equation (3-4b), we can find the susceptibility going forward in cosmological time.

The physical motivation for a decreasing cosmic $\chi(a)$ with increasing scale parameter, " $a$ ", is not known. A possible explanation is as follows. As the scale parameter increases, we can expect more clumping of ordinary matter within regions of space where we have matter. This will lead to higher local temperature in those regions, which in turn implies less local susceptibility, and less polarization, in the surrounding regions. Higher temperature tends to frustrate, and disrupt, any macroscopic ordering of dipole moments. Thus, if the local susceptibility goes down, then the smeared, cosmic average should also start to decrease. There may be other reasons for a decrease in $\chi(a)$ for an increase in scale parameter, but this one comes to mind as being very plausible.

Coming back to our scaling laws, which is given by Equation (3-3a), for dark matter, and, by Equation (3-3b), for dark energy, we are now in a position to come up with an alternative version to Equation (2-6). This version will reflect 
the new assumed scaling behavior. In place of Equation (2-6), we now consider

$$
\begin{aligned}
H^{2}= & 8 \pi G \rho / 3 \\
= & H_{0}^{2}\left[\Omega_{R, 0} a^{-4}+\Omega_{O M, 0} a^{-3}+\Omega_{D M, 0}\left(\chi / \chi_{0}\right)\left(K_{0} / K\right) a^{-3}\right. \\
& \left.+\Omega_{D E, 0}\left(K_{0} / K\right) a^{-3}-\left(\Omega_{0}-1\right) a^{-2}\right]
\end{aligned}
$$

The values for, $\Omega_{R, 0}$, and $\Omega_{D E, 0}$, remain as before. We have broken up, $\Omega_{\text {MATTER }, 0}$, in Equation (2-6), into two components, an ordinary matter component, $\Omega_{O M, 0}$, and a dark matter component, $\Omega_{D M, 0}$, as both now scale differently, as indicated in Equation (3-6). We saw that numerically,

$\Omega_{\text {MATTER }, 0}=0.3106$. We will assume that, $\Omega_{O M, 0}=0.0486$, as indicated by the latest Planck Collaboration [19] [20] [21]. This fixes the dark matter density parameter as, $\Omega_{D M, 0}=(0.3106-0.0486)=0.2620$. We now have all the density parameter coefficients, which are needed for an evaluation of the right hand side of Equation (3-6).

The right hand side of Equation (3-6), also depends on $\chi(a)$, and $K(a)$. We assume Equation (3-4b), holds for, $\chi(a)$, with $\lambda=0.172$. The $K(a)$ is then found using, $K(a)=1-\chi(a)$. The values for, $\left(\chi_{0}, K_{0}\right)=(0.842,0.158)$, are as found in reference [17]. Because the right hand side of Equation (3-6), is a complicated function of scale parameter, "a”, we set up a table and step through various " $a$ " values. The goal is to find that value of scale parameter, $a_{3}$, such that the right hand side of Equation (3-6) vanishes. That is the point where CMB expansion turns into $\mathrm{CMB}$ contraction. This we do next. The results are presented in table form, Table 1.

In Table 1, column 3 is radiative scaling, column 4 represents ordinary matter scaling, column 5 is dark matter scaling, and column 6 reflects dark energy scaling. The curvature scales as in column 8 , and in column 9 , the final column, we have the ratio, $H^{2} / H_{0}^{2}$, as determined by Equation (3-6). One will notice, that

Table 1. Susceptibility decay model.

\begin{tabular}{cccccccc}
\hline$a$ & $\chi(a)$ & $\Omega_{R, 0} a^{-4}$ & $\Omega_{\text {ом,0 }} a^{-3}$ & $\Omega_{D M, 0}\left(\chi / \chi_{0}\right)\left(K_{0} / K\right) a^{-3}$ & $\Omega_{D E, 0}\left(K_{0} / K\right) a^{-3}$ & $\left(\Omega_{0}-1\right) a^{-2}$ & $H^{2} / H_{0}^{2}$ \\
\hline 1 & $8.42 \mathrm{E}-01$ & $9.15 \mathrm{E}-05$ & 0.0486 & $2.62 \mathrm{E}-01$ & $6.91 \mathrm{E}-01$ & $1.802 \mathrm{E}-03$ & $1.000 \mathrm{E}+00$ \\
2 & $7.09 \mathrm{E}-01$ & $5.72 \mathrm{E}-06$ & $6.08 \mathrm{E}-03$ & $1.50 \mathrm{E}-02$ & $4.69 \mathrm{E}-02$ & $4.505 \mathrm{E}-04$ & $6.750 \mathrm{E}-02$ \\
5 & $4.23 \mathrm{E}-01$ & $1.46 \mathrm{E}-07$ & $3.89 \mathrm{E}-04$ & $2.89 \mathrm{E}-04$ & $1.51 \mathrm{E}-03$ & $7.208 \mathrm{E}-05$ & $2.120 \mathrm{E}-03$ \\
10 & $1.79 \mathrm{E}-01$ & $9.15 \mathrm{E}-09$ & $4.86 \mathrm{E}-05$ & $1.07 \mathrm{E}-05$ & $1.33 \mathrm{E}-04$ & $1.802 \mathrm{E}-05$ & $1.743 \mathrm{E}-04$ \\
20 & $3.21 \mathrm{E}-02$ & $5.72 \mathrm{E}-10$ & $6.08 \mathrm{E}-06$ & $2.04 \mathrm{E}-07$ & $1.41 \mathrm{E}-05$ & $4.505 \mathrm{E}-06$ & $1.588 \mathrm{E}-05$ \\
30 & $5.75 \mathrm{E}-03$ & $1.13 \mathrm{E}-10$ & $1.80 \mathrm{E}-06$ & $1.05 \mathrm{E}-08$ & $4.07 \mathrm{E}-06$ & $2.002 \mathrm{E}-06$ & $3.876 \mathrm{E}-06$ \\
40 & $1.03 \mathrm{E}-03$ & $3.58 \mathrm{E}-11$ & $7.59 \mathrm{E}-07$ & $7.91 \mathrm{E}-10$ & $1.71 \mathrm{E}-06$ & $1.126 \mathrm{E}-06$ & $1.342 \mathrm{E}-06$ \\
50 & $1.84 \mathrm{E}-04$ & $1.46 \mathrm{E}-11$ & $3.89 \mathrm{E}-07$ & $7.25 \mathrm{E}-11$ & $8.74 \mathrm{E}-07$ & $7.208 \mathrm{E}-07$ & $5.418 \mathrm{E}-07$ \\
60 & $3.30 \mathrm{E}-05$ & $7.06 \mathrm{E}-12$ & $2.25 \mathrm{E}-07$ & $7.52 \mathrm{E}-12$ & $5.06 \mathrm{E}-07$ & $5.006 \mathrm{E}-07$ & $2.300 \mathrm{E}-07$ \\
70 & $5.91 \mathrm{E}-06$ & $3.81 \mathrm{E}-12$ & $1.42 \mathrm{E}-07$ & $8.48 \mathrm{E}-13$ & $3.18 \mathrm{E}-07$ & $3.678 \mathrm{E}-07$ & $9.229 \mathrm{E}-08$ \\
87.5 & $2.92 \mathrm{E}-07$ & $1.56 \mathrm{E}-12$ & $7.25 \mathrm{E}-08$ & $2.14 \mathrm{E}-14$ & $1.63 \mathrm{E}-07$ & $2.354 \mathrm{E}-07$ & $1.790 \mathrm{E}-10$ \\
87.6 & $2.87 \mathrm{E}-07$ & $1.55 \mathrm{E}-12$ & $7.23 \mathrm{E}-08$ & $2.10 \mathrm{E}-14$ & $1.62 \mathrm{E}-07$ & $2.348 \mathrm{E}-07$ & $-8.972 \mathrm{E}-11$ \\
\hline
\end{tabular}


$H^{2}$ goes from a positive value to a negative value around, $\alpha=87.6$. The critical epoch, $a_{3}$, must, therefore, be close to this value. We have determined the point where expansion turns to contraction for the $\mathrm{CMB}$ radiation. The Hubble radius will have expanded to roughly 87.6 times its current value, and the CMB temperature will drop to approximately, 1/87.6 times its current value, which is, $T_{0}=2.725$.

Why the numbers line up at $a_{3}=87.6$ is unknown. To us, it would seem analogous to asking why water boils at 100 degrees Celsius at atmospheric pressure. It just does. The CMB radiation only has so and so much energy, or stored work, at its disposal for expansion and then contraction. That is why it stops expansion at this temperature, and starts to contract. Looking at Equation (3-1), it is very clear that the final lowest temperature determines the total amount of work done, as well as the final $\mathrm{CMB}$ volume. The steam has run out.

We close with a quick calculation, a crude estimate really, of the efficiency of the Carnot cycle for the CMB radiation, found in WMAP /Planck temperature maps. Using Equation (3-2), we find that

$$
\begin{aligned}
e_{\text {CARNOT }} & =\left(1-T_{C} / T_{H}\right) \\
& =(1-(2.725 / 87.6)(1 / 3.01 \mathrm{E} 27)) \\
& =(1-1.0335 \mathrm{E}-29)
\end{aligned}
$$

We see that the deviation from $100 \%$ efficiency, i.e., $\left(1-e_{\text {CARNOT }}\right)$, is a mere, 1.0335E-29. This heat cycle is extremely efficient. This is a number, for which the significance is unknown, if it even has a special significance. According to Equation (3-17), a closed universe for which $T_{C}$ is allowed to approach a lower value, would lead to an even higher efficiency within this model.

\section{Summary and Conclusions}

We have shown how it is possible to create a closed universe using a cosmic susceptibility model with unconventional scaling behavior for dark matter and dark energy. Utilizing a decreasing value for, $\overline{\chi(a)}$, with an increase in cosmological time, we find that eventually the Hubble constant vanishes, $H=0$ and $H>1$, which would suggest a big bounce from expansion to contraction. Moreover, if we use a specific crude model of decay for $\overline{\chi(a)}$, given by Equation (3-4b), then the universe will start to contract in short order, relatively speaking, i.e., when $a_{3}=87.6$. This surprising value depends on the decay model chosen, and other decreasing susceptibility models can be entertained, which will lead to a different but inevitable outcome, namely, that the universe will eventually reach a point where it will experience a big bounce (crunch) and start to contract.

We have also calculated a specific value for the curvature of space. We believe that in the present epoch, the total curvature reads, $\Omega_{0} \equiv \rho_{0} / \rho_{\text {CRITICAL }, 0}=1.001802$. This is a value well within current, observational bounds, $\Omega_{0}=1.00231_{-0.0054}^{+0.0056}$. This value is so close to unity that one may assume flatness, as in the $\Lambda$ CDM model. We did not do so here. Instead, we assumed that this deviation from flatness is very real. The above value was based on using an established result 
from a previous work [17], namely that the observable Hubble radius equals, $R_{0}=3.217 \mathrm{E} 27$ meters . See Equation (2-12), which relates curvature to radius in the present epoch, assuming a closed universe where, $k=1$.

We built upon our heat engine model for the cosmos. The present voids in the universe, filled with CMB radiation, our thermodynamic "system", follow collectively a Carnot cycle where we have four separate processes. These are isothermal expansion (from point $1 \rightarrow 2$ ), followed by adiabatic expansion (from point $2 \rightarrow 3$ ), followed by isothermal contraction (from point $3 \rightarrow 4$ ), followed by adiabatic contraction (from point $4 \rightarrow 1$ ), bringing us back to our original starting point. See Figure 1. The voids interact with the cooler regions of space, the "surroundings", i.e. exchange heat energy, in the processes from points, $1 \rightarrow 2$, and from point $3 \rightarrow 4$. We have an increase in entropy into the voids from points, $1 \rightarrow 2$, and an increase in entropy into the surroundings from points, $3 \rightarrow$ 4. The two are unequal in magnitude by Equation (3-1). For a closed cycle, we can define a specific amount of work done, which is the area enclosed by the loop in Figure 1. We can also define a specific efficiency, Equation (3-2). The $\mathrm{CMB}$ radiation, we argued, must do actual physical work in expanding and contracting. The heat transfer into the voids, from points, $1 \rightarrow 2$, and the heat transfer out of the voids, from points, $3 \rightarrow 4$, is a necessary ingredient in defining a specific amount of work done. Without it, no work would be possible, as we require an area, which is enclosed by a loop. The isothermal process from points, 1 $\rightarrow 2$, has been identified as the cosmic inflation phase. Following the cycle, and using our crude decay model for cosmic susceptibility, we have found that the deviation from $100 \%$ efficiency is a mere, $1.033 \mathrm{E}-29$, a ridiculously small amount. See Equation (3-7). Whether this result can be tied to other cosmological ratios is unclear.

Further observational work is needed to confirm that the universe is indeed closed. Slight positive curvature $(k=1)$ is assumed but this has yet to be demonstrated. Indeed, we are going very far out on a limb with this assumption. The cosmic susceptibility model, which has been proposed, is also a reach, even though there is some physical motivation from previous work. The specific assumed scaling laws for dark matter and dark energy, Equations (3-3a), and (3-3b), respectively, have to be verified observationally somehow. Finally, other models for cosmic susceptibility decay can be entertained. Our decreasing $\overline{\chi(a)}$ function, with increasing scale parameter value, is but one function out of many, which leads to a very specific outcome. With this particular choice of function, the universe will start to contract already when the universe is 87.6 times current Hubble radius. Other choices for a cosmic susceptibility functional decrease will undoubtedly lead to different bounces, i.e., different turn around points, and new predictions for temperature and volume at point 3.

\section{Acknowledgements}

This work is dedicated to Charmaine Schonblom. 


\section{Conflicts of Interest}

The author declares no conflicts of interest regarding the publication of this paper.

\section{References}

[1] Steinhardt, P. and Ijjas, A. (2018) Bouncing Cosmology Made Simple. Classical and Quantum Gravity, 35, 135004. https://doi.org/10.1088/1361-6382/aac482

[2] Steinhardt, P. and Ijjas, A. (2016) Implications of Planck2015 for Inflationary, Ekpyrotic and Anamorphic Bouncing Cosmologies. Classical and Quantum Gravity, 33, 044001. https://doi.org/10.1088/0264-9381/33/4/044001

[3] Matsui, H., Takahashi, F. and Terada, T. (2019) Bouncing Universe from Nothing. Physics Letters B, 795, 152-159. https://doi.org/10.1016/j.physletb.2019.06.013

[4] Ijjas, A. and Steinhardt, P. (2017) Fully Stable Cosmological Solutions with a Non-Singular Classical Bounce. Physics Letters B, 764, 289-294. https://doi.org/10.1016/j.physletb.2016.11.047

[5] Ijjas, A. and Steinhardt, P. (2016) Classically Stable Nonsingular Cosmological Bounces. Physical Review Letters, 117, 121304. https://doi.org/10.1103/PhysRevLett.117.121304

[6] Brandenberger, R. and Peter, P. (2017) Bouncing Cosmologies: Progress and Problems. Foundations of Physics, 47, 797-850. https://doi.org/10.1007/s10701-016-0057-0

[7] Novello, M. and Bergliaffa, S.E.P. (2008) Bouncing Cosmologies. Physics Reports, 463, 127-213. https://doi.org/10.1016/j.physrep.2008.04.006

[8] Battefeld, D. and Peter, P. (2015) A Critical Review of Classical Bouncing Cosmologies. Physics Reports, 571, 1-66. https://doi.org/10.1016/j.physrep.2014.12.004

[9] Khoury, J., Ovrut, B.A., Steinhardt, P.J. and Turok, N. (2001) The Ekpyrotic Universe: Colliding Branes and the Origin of the Hot Big Bang. Physical Review D, 64, 123522. https://doi.org/10.1103/PhysRevD.64.123522

[10] Buchbinder, E.I., Khoury, J. and Ovrut, B.A. (2008) Non-Gaussianities in New Ekpyrotic Cosmology. Physical Review Letters, 100, 171302. https://doi.org/10.1103/PhysRevLett.100.171302

[11] Graham, P.W., Kaplan, D.E. and Rajendran, S. (2018) Relaxation of the Cosmological Constant. Physical Review D, 97, Article ID: 044003.

[12] Levy, A.M., Ijjas, A. and Steinhardt, P.J. (2015) Scale-Invariant Perturbations in Ekpyrotic Cosmologies without Fine-Tuning of Initial Conditions. Physical Review D, 92, 063524. https://doi.org/10.1103/PhysRevD.92.063524

[13] Levy, A.M. (2017) Fine-Tuning Challenges for the Matter Bounce Scenario. Physical Review D, 95, 023522. https://doi.org/10.1103/PhysRevD.95.023522

[14] Pilot, C. (2018) Modeling Cosmic Expansion, and Possible Inflation, as a Thermodynamic Heat Engine. Zeitschrift fuer Naturforschung A, 74, 1-10. https://arxiv.org/abs/1705.04743

[15] Einstein, A. (1917) Kosmologische Betrachtungen zur Allgemeinen Relativitaetstheorie. [Cosmological Considerations Regarding the General Theory of Relativity.] Königlich Preußische Akademie der. Wissenschaften, Berlin, 142-152.

[16] Wheeler, J.A. (1968) Einstein's Vision. Springer-Verlag, Berlin, Heidelberg and New York, 108.

[17] Pilot, C. (2021) Does Space Have a Gravitational Susceptibility? A Model for the 
Density Parameters in the Friedmann Equation. Journal of High Energy Physics, Gravitation, and Cosmology, 7, 478-507.

https://www.researchgate.net/publication/342993272 Does Space Have a Gravitatio nal Susceptibility A Model for the Density Parameters in the Friedmann Equ ation https://doi.org/10.4236/jhepgc.2021.72028

[18] Pilot, C. (2021) Scaling Behavior for the Susceptibility of the Vacuum. International Journal of Astronomy and Astrophysics, 11, 11-36.

https://www.researchgate.net/publication/342993277 Scaling Behavior for the Su sceptibility of the Vacuum in a Polarization Model for the Cosmos https://doi.org/10.4236/ijaa.2021.111002

[19] Maeder, A., et al. (Planck Collaboration) (2018) Planck 2018 Results. VI. Cosmological Parameters. arXiv:1807.06209 [astro-ph.CO]

[20] Tanabashi, M., et al. (Particle Data Group) (2019) Astrophysical Constants and Parameters (PDF). Physical Review D, 98, 030001.

[21] Ade, P.A.R., Aghanim, N., Armitage-Caplan, C., Arnaud, M., et al. (Planck Collaboration) (2016) Planck 2015 Results. XIII. Cosmological Parameters. Astronomy \& Astrophysics, 594, A13.

[22] Kolb, E.W. and Turner, M.S. (1989) The Early Universe. Addison-Wesley, Reading.

[23] Baumann, D.D. (2015) Lecture Notes on Cosmology. http://theory.uchicago.edu/ liantaow/my-teaching/dark-matter-472/lectures.pdf

[24] Mather, J.C., Fixsen, D.J., Shafer, R.A., et al. (1999) Calibrator Design for the COBE Far-Infrared Absolute Spectrophotometer (FIRAS). The Astrophysical Journal, 512, 511-520. https://doi.org/10.1086/306805

[25] Husdal, L. (2016) On Effective Degrees of Freedom in the Early Universe. Galaxies, 4, 78. https://doi.org/10.3390/galaxies4040078 